\title{
Characterization of Adenylate Cyclase Interacting Protein ACI1 in the Rice Blast Fungus, Magnaporthe oryzae
}

\author{
Jixin Deng ${ }^{1,2}$ and Ralph A. Dean ${ }^{* 1}$ \\ ${ }^{I}$ Center for Integrated Fungal Research, North Carolina State University, Raleigh, NC 27695, USA \\ ${ }^{2}$ Human Genome Sequencing Center, Baylor College of Medicine, Houston, TX 77030, USA
}

\begin{abstract}
Host infection by Magnaporthe oryzae requires the formation of a specialized infection structure, the appressorium. Using yeast 2-hybrid, ACI1 was found to interact with $M A C l$, a key gene in the cAMP signaling pathway regulating appressorium formation. Targeted ACI1 gene replacement mutants exhibited significantly delayed and slightly lower levels of appressorium formation, but were indistinguishable from the wild type strain in terms of pathogenicity. No differences were observed in colony morphology and wettability, growth rate, conidiation and salt tolerance. These results suggest that the ACI1 is required for normal appressorium formation, but not for pathogenicity and vegetative growth. Characterization elsewhere of an ACI1 homologue IMR gave similar results. Functional redundancy resulting from the presence of related homologues in the M. oryzae genome is one explanation that may account for these findings.
\end{abstract}

Keywords: Rice blast, adenylate cyclase, signal transduction, appressorium, pathogenicity.

\section{INTRODUCTION}

The rice blast fungus, Magnaporthe oryzae is a major threat to rice production. To infect rice or other grass hosts, the fungus develops a specialized infection structure, the appressorium. Signal transduction pathways, in particular, the cyclic AMP (cAMP) and MAPK response pathways play a crucial role in regulating infection structure formation [1, 2]. Many components of these pathways have been identified and characterized over the past decade. For the cAMP pathway, two key pathway component genes, adenylate cyclase gene $(M A C 1)$ [3] and catalytic subunit of PKA gene (CPKA) [4], were found to be required for appressorium morphogenesis and pathogenicity. Analyses of other possible pathway components such as hydrophobin gene (MPG1) [5], G protein coupled receptor gene (PTH11) [6] and $\alpha$ subunit of a heterotrimeric $\mathrm{G}$ protein $(M A G B)$ [7] provide additional supporting evidence for these signal pathways. However, the specific mechanisms of how the products of these genes participate in these pathways remains to be elucidated. For example, MPG1 appears to be involved in host surface interaction during infection process and may act as an upstream sensor for appressorial formation. A possible candidate downstream of MPG1 in the cAMP pathway is PTH11. Recently, PTH11 was characterized as a member of a novel class of G protein coupled receptors (GPCRs) by in-silico studies [8]. Therefore, it is possible that PTH11 perceives a signal from MPG1 following interaction with the host surface, however, there is no direct evidence. PTH11 is presumed to interact with $\mathrm{G}$ proteins. M. oryzae genome contains three $\alpha$ subunits, two $\beta$ subunits and one $\gamma$ subunit of the heterotrimeric $\mathrm{G}$ protein. Three $\alpha$ subunits have been characterized in M. oryzae, but only MAGB mutants exhibit

\footnotetext{
*Address correspondence to this author at the Center for Integrated Fungal Research North Carolina State University, Raleigh, NC 27695, USA;

Tel: 919-513-0020; Fax: 919-513-0024; E-mail: ralph_dean@ncsu.edu
}

a dramatic effect on appressorium formation [7]. However, again there is no experimental evidence demonstrating MAGB directly interacts with PTH11. In animal hormone activated signal transduction pathways, dissociation of $\alpha$ subunit of $\mathrm{G}$ protein from $\beta \gamma$ heterodimer is stimulated by a conformation change of GPCR and the freed $\alpha$ subunit then binds and activates adenylate cyclase. The application of this model to MAGB has not been established in M. oryzae; there is no data showing direct binding of the $\alpha$ subunit to MAC1. In contrast, in M. oryzae, it was suggested that the G $\beta \gamma$ heterodimer may be a repressor of adenylate cyclase activity and repression is released when $\alpha$ subunit is bound to $G \beta \gamma$ heterodimer under certain conditions [9]. To directly identify other interacting components of cAMP pathway, especially those involved in the early events, MAC1 and CPKA were used as bait in yeast 2-hybrid assays [10]. One gene that was identified multiple times by MACl fragments was $A C I 1$. ACI1 shares a novel fungal specific CFEM domain with PTH11, however, the function of this domain is unknown. Like PTH11, ACI1 also has predicted trans-membrane spans suggesting it is a possible membrane protein. To determine whether ACI1 is involved in the signal cascade regulating appressorium formation and pathogenicity, the gene was deleted by targeted mutagenesis. We report here that deletion mutants exhibited delayed and reduced appressorium formation, however, there was no significant change in virulence. The presence of possible homologs of ACI1 in M. oryzae with similar expression profiles is discussed in the context of functional redundancy and/or other compensatory process.

\section{MATERIALS AND METHODS}

\section{Strains and Growth Conditions}

M. oryzae strain 70-15 (MAT1-1) was used throughout all experiments. Wild type and all the transformants were cultured on oatmeal agar plates $(50 \mathrm{~g}$ of oatmeal per liter) at $25^{\circ} \mathrm{C}$ under fluorescent light for conidiation. Conidia were collected from 10-day-old cultures and washed twice with 
distilled water for further inoculation. Medium used for growing mycelia was CM (yeast extract, 6g; casein acid hydrolysate, $6 \mathrm{~g}$; sucrose, $10 \mathrm{~g}$; per liter).

\section{Genomic DNA and Appressorial RNA Isolation}

A previously described method [11] was followed with some modifications to isolate genomic DNA of M. oryzae. For appressorium RNA preparation, appressoria were induced by inoculating $100 \sim 200 \mathrm{ml}$ of conidium suspension $\left(1 \times 10^{6}\right.$ conidia/ml in distilled water) on an inductive surface of cellophane membranes (Promega) for $4-6 \mathrm{~h}$ at $25{ }^{\circ} \mathrm{C}$. After the liquid was poured off, the cellophane membrane with the appressoria attached was ground into a fine powder using liquid nitrogen. Total RNA was isolated using the $\mathrm{LiCl}$ method [12].

\section{Construction of Targeted Gene Disruption Vector}

A gene disruption vector was constructed using the pCB1004 vector [13] containing an $h p h$ gene expression cassette. A $2 \mathrm{~kb} \mathrm{5}$ ' genomic fragment and a $2 \mathrm{~kb} 3$ ' genomic fragment of ACI1 gene was amplified by PCR with engineered restriction sites at 5' and 3' side of each fragment. Primers to amplify 5' fragment and 3' fragments are ACI55Bam, ACI5-3Sac, ACI3-5Kpn and ACI3-3Cla (Table 1). The PCR product of 3' fragment was digested with KpnI and $\mathrm{ClaI}$ and ligated into double digested pCB1004 vector to create pCB1004-ACI1-R. Similarly, the PCR product of the 5 ' fragment was digested with BamH1 and SacI and ligated into the correspondingly double digested pCB1004-ACI1-R to create pCB1004-ACI1. pCB1004-ACI1 was linearized by EcoRI and purified by gel electrophoresis for fungal transformation.

\section{Fungal Transformation}

Protoplast preparation from M. oryzae and DNAmediated transformation were performed following established procedures with slight modification [14]. Transformants were transferred to V8 juice agar plates supplemented with hygromycin $(100 \mathrm{ug} / \mathrm{ml})$ for selective growing. Initial identification of gene disruption mutants was performed by PCR. PCR amplification of a $2 \mathrm{~kb}$ product using primers $\mathrm{P} 1-5$ and P1-3 (Table 1) was expected in transformants that had undergone homologous recombination between CB1004$\mathrm{ACI} 1$ and the ACI1 genomic sequence (Fig. 1a). Integration of $h p h$ gene, expression cassette was also confirmed by PCR with $h p h$ specific primers $\mathrm{P} 0-5$ and $\mathrm{P} 0-3$ (Table 1). Transformants were purified by single-spore isolation and used for further analyses.

\section{Southern and Northern Blotting and Hybridization}

Southern blots were prepared using total genomic DNA digested to completion, separated by electrophoresis on $1 \%$ TAE agarose gel and transferred to Nylon membranes following standard procedures. For the Northern blots, total RNA was dissolved in denaturing solution [12], incubated at $65^{\circ} \mathrm{C}$ for 20 mins and chilled on ice. Denatured RNA were separated on a $1 \%$ TAE agarose gel containing $2.2 \mathrm{M}$ formaldehyde and blotted onto Nylon membranes. Standard conditions for prehybridization, hybridization, and washing were standard used [15]. Two probes were used for both KpnI and HindIII digested genomic DNA Southern blots. For the first probe, a PCR product for 3' fragment of ACI1 gene using ACI3-5Kpn and ACI3-3Cla primer pairs was obtained. The PCR product was double digested with KpnI and HindIII enzyme and the resulting $1.5 \mathrm{~Kb}$ fragment gel purified using QIAGEN kit. To prepare the second probe, a PCR product of the $h p h$ gene using PCB1004 as template and PCR primers $\mathrm{P} 0-5$ and $\mathrm{P} 0-3$, was gel purified. To prepare an ACI1 gene probe for Northern blots, the insert was cut from the appressorial stage cDNA clone, (Genbank AI068796), with EcoRI and XhoI and gel purified.

\section{Appressorium Formation Assay}

Conidia were collected from 5-7 days old oatmeal agar cultures in sterile distilled water and adjusted to $10^{4}$ conidia per $\mathrm{ml}$. Fifty ul conidial suspension drops were placed on hydrophobic side of gelbond and incubated in a moist chamber at room temperature. The number and percent of germinated conidia and those that elaborated appressoria were examined at 2, 4, 6, 8, 10, 12 and 24 hours after incubation. The experiment was repeated three times with three replicates. Student's t-test was used to evaluate differences in germination and appressorium formation between strains.

\section{Plant Infection Assays}

For plant infection assays, both susceptible rice cultivars and barley cultivars were used. Twelve-day-old rice seedlings were grown on agar media in glass tubes $(20 \mathrm{~cm}$ long and $5 \mathrm{~cm}$ in diameter). To obtain larger plants, rice and barley were grown on composite soil in the greenhouse for 15

Table 1. List of PCR Primers Used

\begin{tabular}{|c|c|l|}
\hline Name & Engineered Restriction Sites & \multicolumn{1}{c|}{ Sequence } \\
\hline \hline ACI5-5Bam & BamH1 & 5'-GCG GAT CCT ACG GCC ATG AGT CAC TTT G-3' \\
\hline ACI5-3Sac & SacI & 5'-GGG AGC TCA GCC AAG ATC AAG CAA GTG T-3' \\
\hline ACI3-5Kpn & KpnI & 5'-CGG GTA CCG ACC GCC AAC GTC TAC TTT C-3' \\
\hline ACI3-3Cla & ClaI & 5'-CCA TCG ATA TGC TCG AAC CCA TCT GC-3' \\
\hline P0-5 & n.a & 5'-TCT CGTGCT TTC AGC TTG G-3' \\
\hline P0-3 & n.a & 5'-GGC GTC GGT TTC CAC TAT C-3' \\
\hline P1-5 & n.a & 5'-CAC CGA GAT GCT TGT TTG AC-3' \\
\hline P1-3 & n.a & 5'-GGC CTT TTG CTC ACA TGT TC-3' \\
\hline
\end{tabular}

n.a: Not applicable. 
(a)
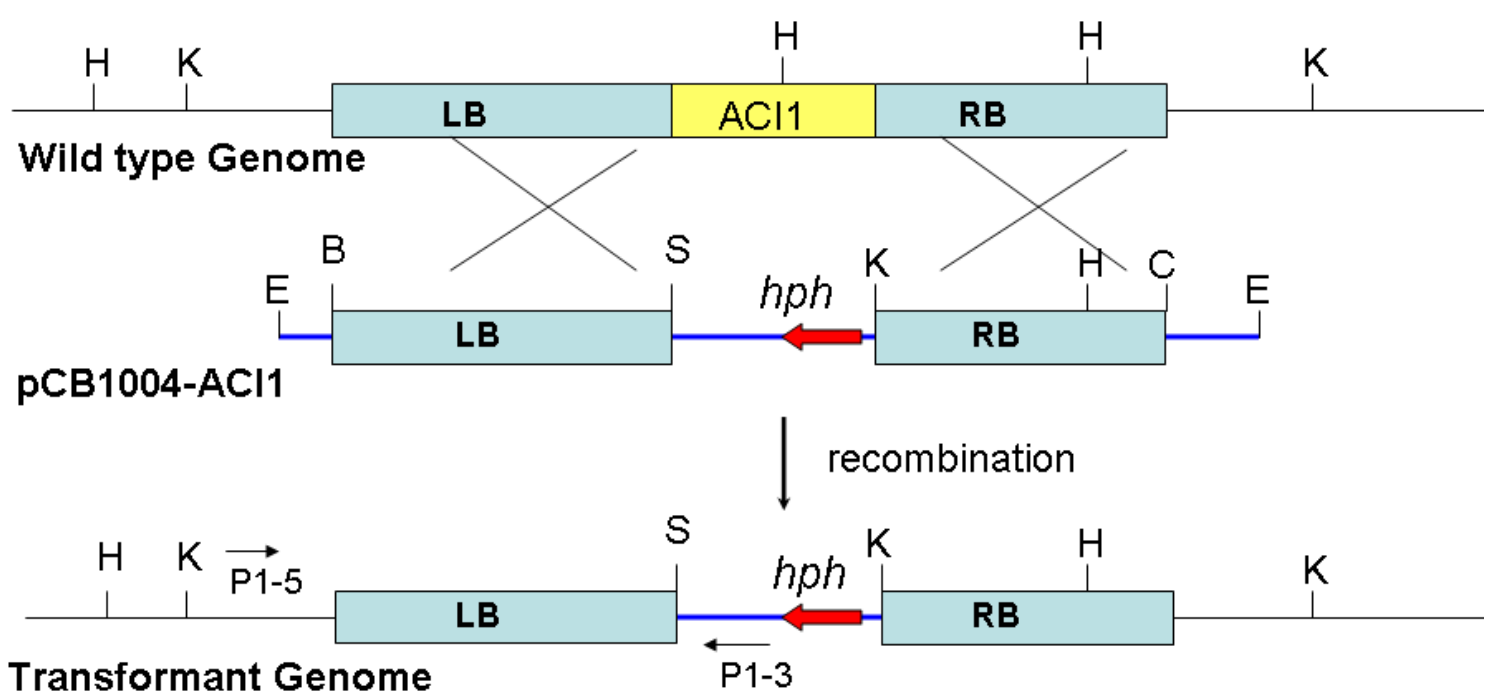

Transformant Genome

(b)

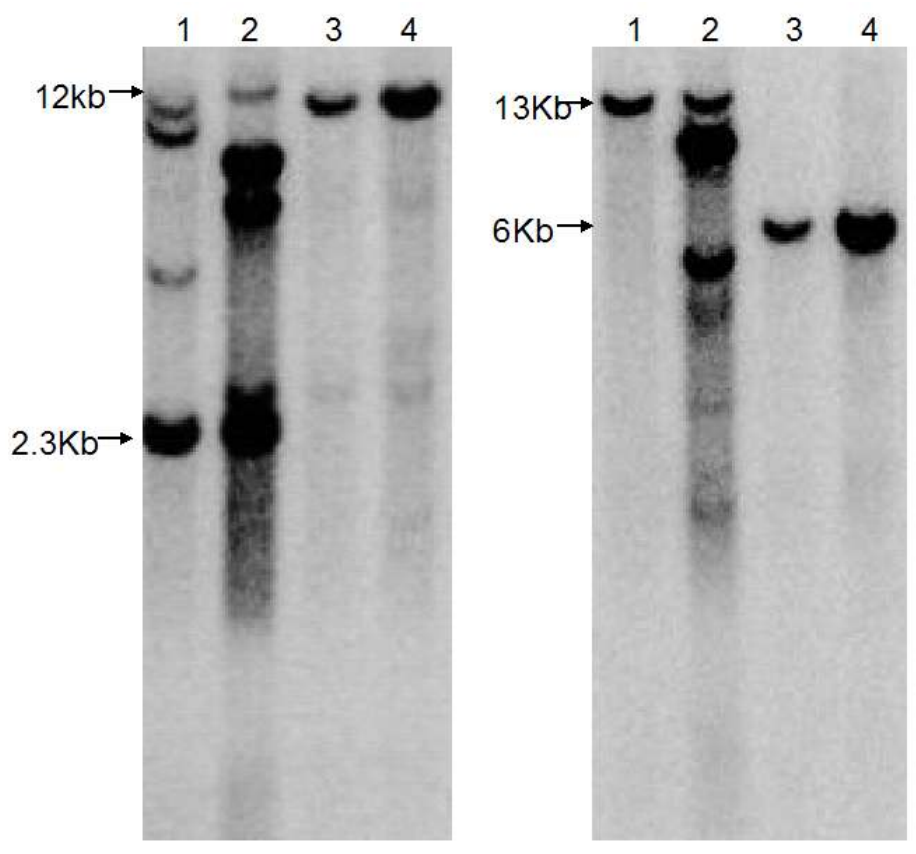

Fig. (1). Deletion of ACI1 by double homologous recombination. (a) Schematic diagram for deletion of ACI1. A gene replacement cassette was constructed by flanking the hph gene with left $\left(5^{\prime} \mathrm{LB}\right)$ and right $(3$ 'RB) boarder sequence from ACI1. The resulting construct, PCB1004ACI1 was linearized with EcoRI and transformed into M. oryzae. H represents HindIII restriction site; K represents KpnI. P1-5 and Pl-3 represent binding sites for specific primers used in the initial screen of transformants. (b) Southern blot analysis of transformants. Left panel; genomic DNA restructed with HindIII. Right panel; genomic DNA restricted with KpnI. Lane 1, wild type; lane 2 ACI7; lane 3 ACI1 7; lane 4 ACI24.

days before inoculation. For preparing fungal materials, conidia were suspended at a concentrations of $\sim 10^{5}$ conidia $/ \mathrm{ml}$ in $0.05 \%$ Tween 20 with distilled water. Five $\mathrm{ml}$ conidial suspension was evenly sprayed onto the test plants with a fine mist aspirator. Inoculated plants were inoculated in a growth chamber at $25^{\circ} \mathrm{C}$ in the dark for the first 24 , followed by a $12 / 12$ light/dark cycle enclosed in plastic to keep humidity high. Lesions were observed daily and photographed after one week. The experiment was repeated three times with three replicates.

\section{Growth Rate and Osmosensitivity Assay}

Small (3mm diameter) agar blocks at the edge of 10 - to 14- day-old cultures were cut out as uniform circles and placed onto the center of a freshly prepared medium (complete medium and minimum medium) in Petri plates. For the osmosensitivity assay, $0.4 \mathrm{M} \mathrm{NaCl}$ was supplemented in each plate. Cultures were incubated at $20^{\circ} \mathrm{C}$ with constant fluorescent light. The diameter of each colony was examined every day from day 2 lasting for 6 days. Hyphal morphology was observed daily. Each experiment contained three replicates, which was repeated twice. 


\section{Wettability Assay}

Twenty drops of 50ul sterile distilled water were placed on the surface of 8-10 day old fungal cultures grown on oatmeal plates. The plates were incubated for $12 \mathrm{~h}$. Thereafter, the number of drops that had soaked into the surface was counted. Three plates were used as replicates.

\section{Sequence Analyses}

ACI1 gene is listed in Genbank under accession number AAN64312.1 for protein sequence and AY166602.1 for DNA sequence. The gene number in Magnaporthe oryzae Database

(http://www.broad.mit.edu/annotation/fungi/magnaporthe/) is MG05531. Interproscan (ver 4.2 Release 12.1) was run on ACI1 protein sequence to identify functional domains. Possible homologs of ACI1 gene which contained the CFEM domain were searched by querying Genbank using ACI1 protein sequence and BlastP with a cutoff E-value of 0.1. Retrieved protein sequences were aligned by T-Coffee [16].

\section{RESULTS}

\section{Functional Domain Predictions in ACI1}

ACI1 was previously annotated as 722 nucleotides and 184 amino acid long, with three exons [10]. Further analysis carried out here using Interproscan identified four possible functional domains; a CFEM domain; a signal peptide sequence; a transmembrane region and a scorpion toxin-like domain. The transmembrane region overlapped partially with the CFEM domain, which was predicted as an extracellular domain [17]. The CFEM domain was previously proposed to be associated with pathogenicity [17]. The presence of both the signal peptide sequence and transmembrane region indicated that ACI1 is possibly a membrane located protein. This was also supported by the detection of a GPIanchor site at the C-terminal of the protein based on analyses using Big-PI [10]. In addition, the scorpion toxin domain is involved in binding membrane receptors in mammals. Overall, these features combined with the yeast two hybrid data, supports the hypothesis that ACI1 acts as a membrane receptor or receptor associated protein involved in cAMP signal transduction pathway.

\section{Target Disruption of $\boldsymbol{A C I I}$}

To determine the function of ACI1, a targeted gene replacement strategy was employed. The gene disruption vector was constructed by inserting $\sim 2 \mathrm{~kb}$ left and right broader fragments flanking $A C I 1$ into the vector pCB1004 [13]. The resulting disruption vector, pCB1004-ACI1 (Fig. 1a) contained the hygromycin resistance gene $(h p h)$ cassette flanked by fragments of $A C I 1$. Based on PCR screening of hygromycin-resistant colonies, two colonies (ACI17 and ACI24) were identified as deletion mutants. These results were confirmed by two Southern hybridization analyses. First, a $1.5 \mathrm{~kb}$ ACI1 3 ' flanking fragment was used as a probe (see methods) and hybridized with HindIII digested genomic DNA. Wild type and a randomly selected ectopic transformant ACI7 revealed a $2.3 \mathrm{~kb}$ band as expected, while knockout mutants ACI17 and ACI24 both yielded a 12-kb fragment instead (Fig. 2b). Secondly, the same probe when hybridized with KpnI digested genomic DNA resulted in $6 \mathrm{~kb}$ fragments in both knockout mutants while the wild type and ectopic strains identified $13 \mathrm{~kb}$ fragments as expected (Fig. 2b). The randomly selected ectopic ACI7 showed several bands in both Southern experiments, indicating multiple integration events. Northern blot analyses was further undertaken to confirm deletion of the ACII gene in the two mutant strains. No expression of ACI1 was found in mutant strains (data not shown). Overall, our results support the conclusion that both transformants ACI17 and ACI24 underwent homologous recombination events replacing $A C I l$ with the hygromycin gene cassette.

\section{Appressorium Formation is Delayed in ACI1 Deletion Mutants}

To characterize whether ACI1 was required for the rice blast fungus to germinate and form appressoria, a time course experiment was employed using the wild type, ectopic and mutant strains (Table 2). Very little differences were observed for the rate and overall level of conidia germination. Two hours after being placed on the hydrophobic surface of Gelbond, most conidia of all strains had germinated but very few appressoria had formed. At 4 hours after inoculation, $\sim 20 \%$ of the germinated conidia of the wild type and ectopic strains had formed appressoria while only $\sim 2 \%$ $(\mathrm{P}<0.001)$ were produced by the mutant stains. Appressorium formation by mutants remained significantly $(\mathrm{p}<0.05)$ lower than the wild-type and ectopic strains through 24 hours, however, absolute differences were small. At 24 hours, the last time point data were collected, $\sim 80 \%$ of conidia formed mature appressoria in mutant strains compared to $\sim 86 \%$ in wild-type and ectopic strains.

\section{ACI1 is Dispensable for Pathogenicity}

To test whether $A C I 1$ was required for pathogenicity, $A C I 1$ null mutants were compared with the ectopic and wild type strains for their ability to cause disease using several infection assays. Assays were performed on small rice seedlings, which were grown in small glass tubes, and on rice seedlings that were grown under greenhouse conditions. No clear symptom differences between mutant strains and other strains were observed on tube grown plants after one week after incubation (data not shown). Similar results were obtained using greenhouse grown rice seedlings, which were about $30 \mathrm{~cm}$ tall when assayed. Wild type, ectopic and mutant strains all caused diamond-shaped lesions and the density of lesions was similar among all strains (Fig. 2a). Finally, there was no clear difference in the ability to cause disease symptoms on barley (Fig. 2b). In summary, ACI1 does not seem to be required for pathogenicity on susceptible hosts.

\section{ACI1 is Not Essential For Mycelial Growth Under Tested Conditions}

Growth rate on $\mathrm{CM}$ media, $\mathrm{MM}$ media, $\mathrm{CM}+\mathrm{NaCl}$ media, colony diameters were measured every day and compared among all strains. Under all tested conditions, including high salt conditions, we were unable to detect significant differences in growth rate (data not shown). In addition, no difference in colony morphologies were observed, indicating that ACI1 is not essential for growth and colony morphology in culture. 
(a)

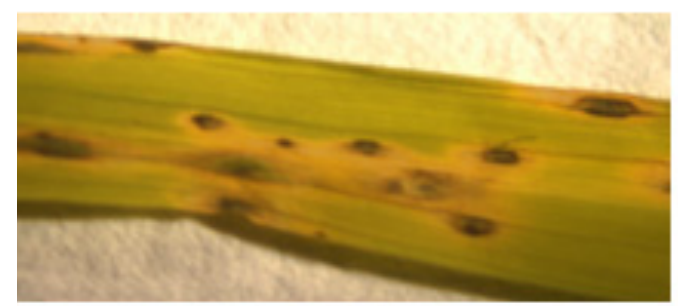

wht

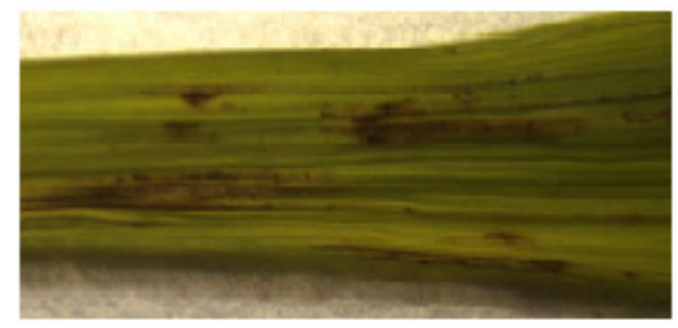

$\mathrm{ACl17}$

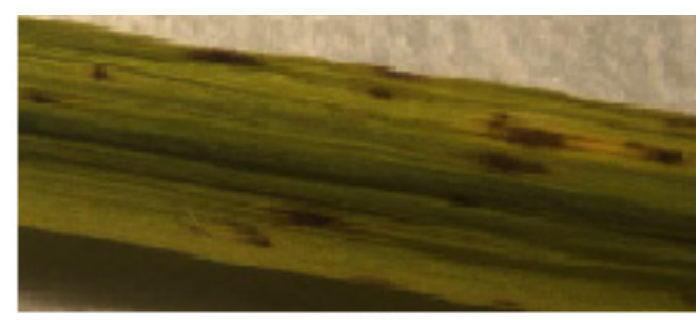

$\mathrm{ACl7}$

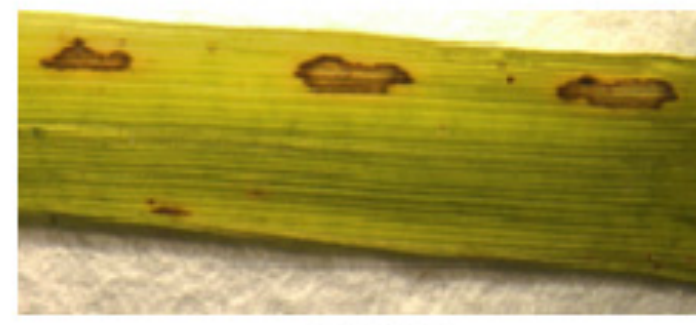

$\mathrm{ACl} 24$

(b)

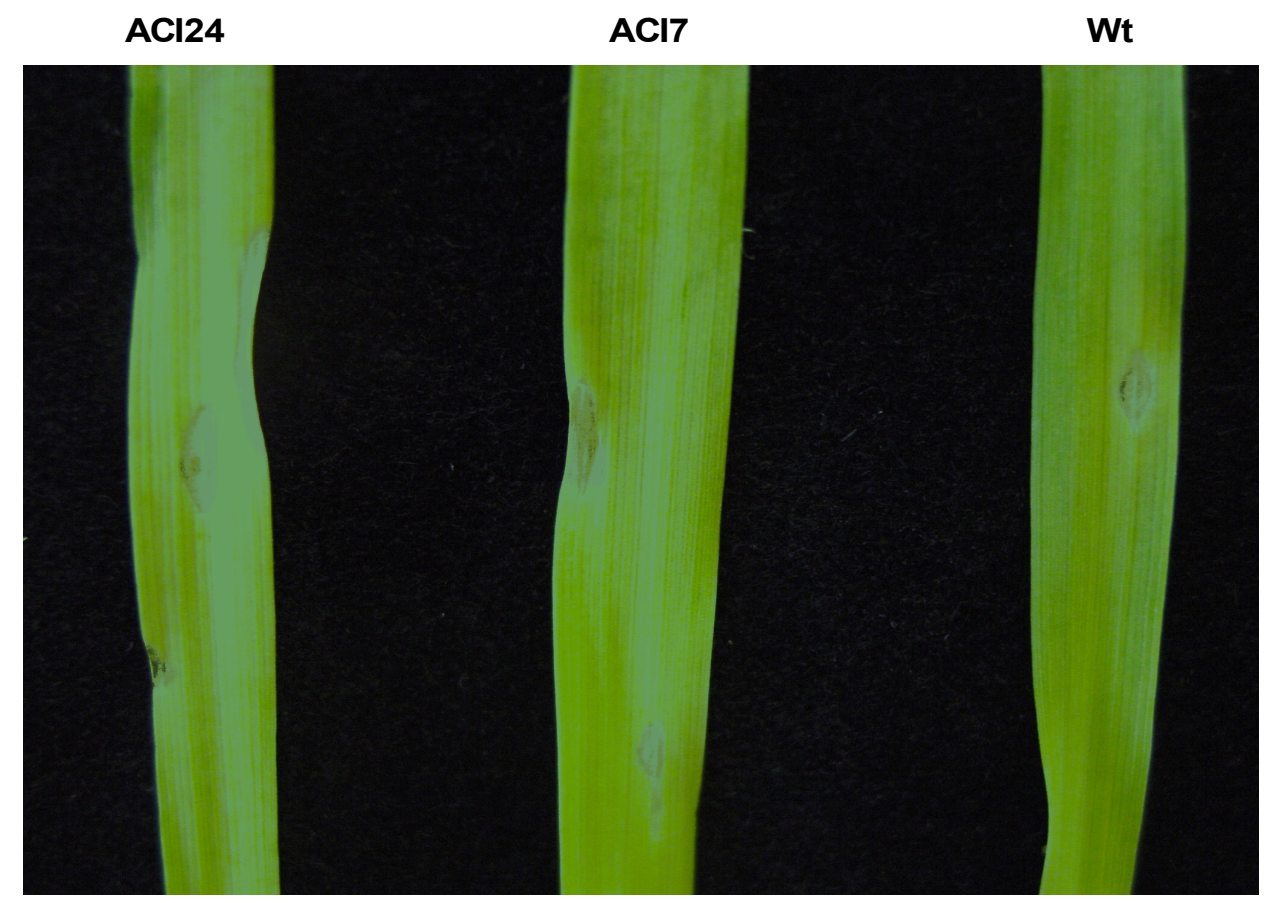

Fig. (2). Pathogenicity assays with ACI1 deletion strains. (a) Rice leaves inoculated with deletion strains ACI17 and ACI24, ectopic strain ACI7 and wild-type (Wt). (b) Infection assay on barley leaves. Disease symptoms were observed 7 days after inoculation.

\section{ACI1 Mutants Did Not Show Easily Wettable Phenotype}

An easily wettable phonotype has been reported for several mutants defective in fungal hydrophobins [18-20]. Based on the predicted signal peptides and extracellullar domains, we were interested to know whether the CFEM domain was related to hydrophobin function. To test this, water droplets were placed on colonies and the time for the droplets to be absorbed was measured. No differences were noted compared to wild type and ectopic strains indicating that the easily wetting phenotype was not observed in $A C I 1$ deletion mutants under our experimental conditions, suggesting ACI1 does not share this property with hydrophobins.

\section{Possible Homologues of $A C I 1$ Gene in M. oryzae Genome}

Through BlastP analyses (E-value cutoff $<0.1), 16$ possible homologs of ACI1 were retrieved from Genbank (Table 3 ), one of these (XP 368095) found in the genome sequence of $M$. oryzae strain 70-15 was identical to a putative immunoreactive protein IMR previously characterized in the P2 strain of $M$. oryzae [21]. The most closely related homolog of ACI1 was XP_964641 from Neurospora crassa. As shown in Fig. (3), both sequences aligned over most of their length suggesting they may represent an orthologous pair, however, sequence identity was quite low (38\% amino acid identity). 
Table 2. Comparison of Conidial Germination and Appressorium Formation Among Wild Type, Ectopic and ACI1 Mutant Strains

\begin{tabular}{|c|c|c|c|c|}
\hline \multirow{2}{*}{ Time } & \multicolumn{2}{|c|}{ Wild Type + Ectopic (ACI7) } & \multicolumn{2}{|c|}{ Mutants (ACI17 + ACI24) } \\
\cline { 2 - 5 } & Germ $^{\text {a }}$ & App $^{\text {b }}$ & Germ & App \\
\hline \hline 2h & $76.6 \pm 9.5$ & $3.5 \pm 3.4$ & $74.0 \pm 7.2$ & $0.3 \pm 0.9^{*}$ \\
\hline 4h & $79.0 \pm 7.5$ & $21.0 \pm 5.2$ & $76.3 \pm 3.1$ & $2.3 \pm 1.7^{* * *}$ \\
\hline $6 \mathrm{~h}$ & $82.3 \pm 7.1$ & $70.8 \pm 9.7$ & $76.5 \pm 4.6$ & $54.2 \pm 9.0^{*}$ \\
\hline $8 \mathrm{~h}$ & $91.0 \pm 3.3$ & $81.0 \pm 3.7$ & $83.3 \pm 4.9^{\dagger}$ & $67.7 \pm 8.5^{* *}$ \\
\hline $12 \mathrm{~h}$ & $90.7 \pm 3.7$ & $84.7 \pm 2.2$ & $88.5 \pm 3.0$ & $77.0 \pm 6.4^{*}$ \\
\hline $24 \mathrm{~h}$ & $91.7 \pm 2.6$ & $86.5 \pm 2.8$ & $89.5 \pm 1.5$ & $79.8 \pm 4.7^{*}$ \\
\hline
\end{tabular}

${ }^{\mathrm{a}}$ Percentage of germinated conidia

${ }^{\mathrm{b}}$ The percentage of germinated conidia with appressoria.

*******Represents significant difference between ACI deletion mutants (ACI17 and ACI24) and Wild type and Ectopic ACI7 for appressorium formation based on t-test at $\mathrm{P}<0.05, \mathrm{P}<0.01$ and $\mathrm{P}<0.001$ respectively.

${ }^{\dagger}$ Represents significant difference between ACI deletion mutants (ACI17 and ACI24) and Wild type and Ectopic ACI7 for germination based on t-test at $\mathrm{P}<0.05$.

In addition to IMR, only one other possible homolog (XP 364725) of ACI1 was identified in M oryzae. Both sequences showed low sequence identity with ACI1. Compared to ACI1, IMR has $19 \%$ amino acid identity whereas XP 364725 has $15 \%$ amino acid identity. Inspection of the alignment (Fig. 3) revealed that sequence matches were primarily in the CFEM domain region with rarely a few amino acids extension on both ends. The matched amino acids in the CFEM domain was sparsely distributed, essentially corresponding to the consensus motif sequence of CFEM, $\mathrm{PxC}[\mathrm{A} / \mathrm{G}] \mathrm{x} 2 \mathrm{Cx} 8-12 \mathrm{Cx} 1-3[\mathrm{x} / \mathrm{T}] \mathrm{Dx} 2-5 \mathrm{CxCx} 9-14 \mathrm{Cx} 3-4 \mathrm{Cx}$ 15-16C [17]. Therefore, it appears that there are no close homologs of ACI1 in the rice blast fungal genome and that the region of similarity is restricted to the CFEM motif. Southern blots analyses under moderate stringency using ACI1 gene as a probe were consistent with the in-silico analyses. Only a single band was found in the genome of wild type and ectopic strains and no bands were evident in the genome of deletion mutants (data not shown).

\section{DISCUSSION}

Previous studies demonstrated that MAC1 is required for a variety of physiological processes in the rice blast fungus including conidiation, conidial germination, vegetative growth, appressoria formation and virulence [3]. MAC1 is an essential component for cAMP signaling. The physical interaction of ACI1 and MAC1 in previous yeast two-hybrid studies suggested the possible role of ACI1 in cAMP signaling and presumably, in the physiological processes involving the $M A C l$ gene. Moreover, a novel fungal specific domain CFEM [17] spanning amino acid 28 to 92 was found to be present in ACI1. This domain was also found in PTH11, which is known to play a key role in signal sensing during appressorium development and pathogenicity. PTH11 and ACI1 both contain signal peptides. Furthermore, ACI1 contains predicted GPI anchor sites, suggesting that the protein has a membrane or extracellular location. The presence of a domain similar to the one found in the scorpion toxin is intriguing. In mammals, this domain is involved in binding and inhibiting sodium channels, thus blocking neural transmission. It is possible that this type of domain in $M$. oryzae may also be involved in binding to membrane receptors. Therefore, ACI1 possesses several potential important properties that could serve to communicate external signals to the intracellular adenylate cyclase. To test this hypothesis, we first assayed the phenotype changes of ACI1 deletion mutants

Table 3. Possible Homologs of ACI1 in M. oryzae and Other Fungal Genomes

\begin{tabular}{|c|c|c|}
\hline Protein Accession $^{\mathrm{a}}$ & Description & E-Value \\
\hline ref|XP_964641.1| & hypothetical protein [Neurospora crassa OR74A] & $2 \mathrm{e}-16$ \\
\hline ref|XP_364725.1| & predicted protein [Magnaporthe grisea 70-15] & $1 e-10$ \\
\hline ref|XP_001595694.1| & hypothetical protein SS1G_03783 [Sclerotinia sclerotiorum 1980] & $8 \mathrm{e}-04$ \\
\hline ref|XP_660049.1| & hypothetical protein AN2445.2 [Aspergillus nidulans FGSC A4] & 0.001 \\
\hline gb|EAT77550.1| & predicted protein [Phaeosphaeria nodorum SN15] & 0.001 \\
\hline ref|XP_382331.1| & hypothetical protein FG02155.1 [Gibberella zeae PH-1] & 0.004 \\
\hline gb|EAT82875.1| & hypothetical protein SNOG_09610 [Phaeosphaeria nodorum SN15] & 0.011 \\
\hline gb|EAT87006.1| & hypothetical protein SNOG_05942 [Phaeosphaeria nodorum SN15] & 0.012 \\
\hline ref|XP_368095.1| & conserved hypothetical protein [Magnaporthe grisea 70-15] & 0.029 \\
\hline dbj|BAC65876.1 & putative immunoreactive protein [Magnaporthe grisea] & 0.029 \\
\hline ref|XP_382550.1| & hypothetical protein FG02374.1 [Gibberella zeae PH-1] & 0.034 \\
\hline gb|AAC49140.1| & immunoreactive spherule cell wall protein & 0.043 \\
\hline ref|XP_001240075.1| & hypothetical protein CIMG_09696 [Coccidioides immitis RS] & 0.064 \\
\hline ref|XP_001219512.1| & predicted protein [Chaetomium globosum CBS 148.51] & 0.073 \\
\hline ref|XP_958559.1| & hypothetical protein [Neurospora crassa OR74A] & 0.083 \\
\hline ref|XP_385997.1| & hypothetical protein FG05821.1 [Gibberella zeae PH-1] & 0.098 \\
\hline
\end{tabular}




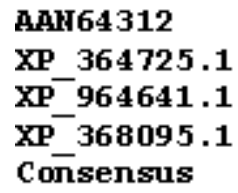

AAH64312 XP 364725.1 XP-964641.1 XP-368095.1 consensus

\section{AAH64312 XP 364725.1 XP-964641.1 XP_368095.1 consensus}

\section{AAH64312 XP 364725.1 XP- 964641.1 XP-368095.1 consensus}

\section{AAH64312 XP 364725.1 XP-964641.1 XP 368095.1 consensus}

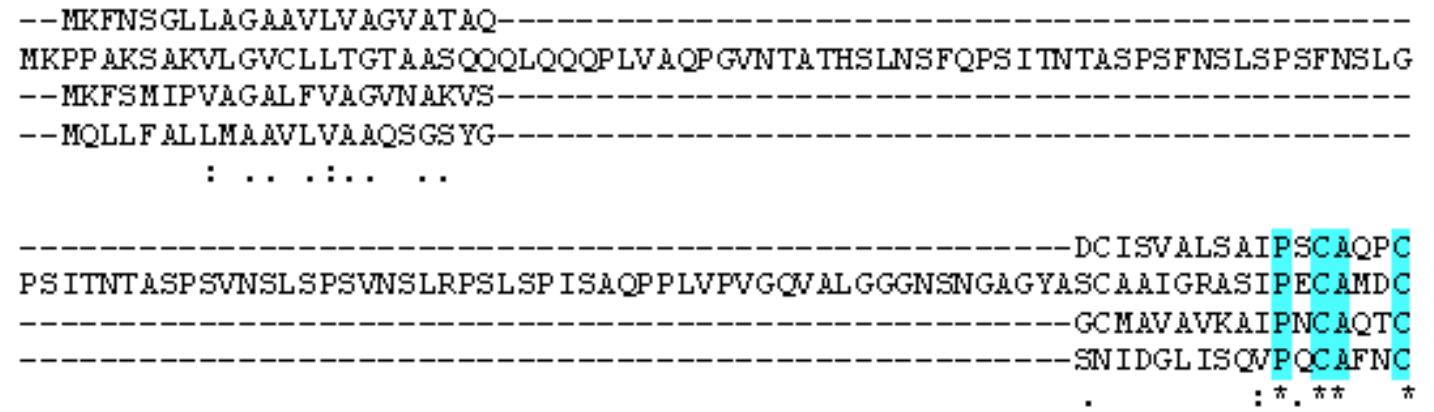

FLNG AP T IGCSGTDF KCQCQQQAKMF A AVESCVQKSCPESEF QKT IDGSDKVCT-C ASGGP ASNNAGGAG

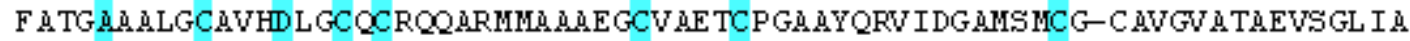

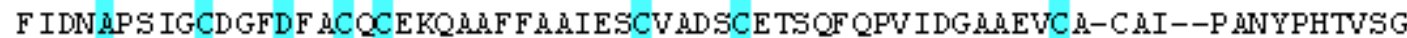
LAEẢ ÄESSCGL TD IRCMCGRISLVSGTSSTCLSKACS ADQL A TLSST ISKVC ADVVGA------------

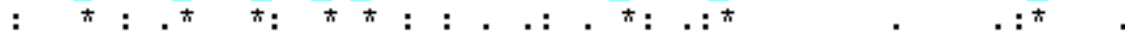

NTVNP----SSF IPGPTSTẢSPTTTVẢP TGT--------------------PSGRPS AVP TẢ------R-----------PGPTGTÁSVS ASYSYMSGSGF SVVSSLTVINKẢ ASGTẢ TPSFMPQVDQPSQMVP IHK TVVPPKGTVSSIVPCPTTIGS---------VIPSGI ASA YP TLSE---GYP I ASEMPGASSTSAKPP---

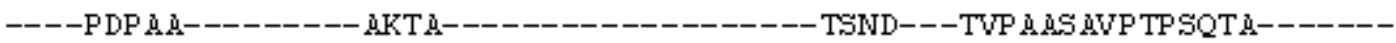
: . $\quad$ * . :

Fig. (3). Sequence alignment of ACI1 with its closest homologs in M. oryzae and N. crassa. AAN64312 is the GenBank accession number for ACI1; XP_964641.1 is the N. crassa homolog; XP_364725.1 is a M. oryzae homolog; XP_368095.1 is IMR from M. oryzae. The shaded columns indicate conserved residues in the CFEM domain.

that were shown to be associated with the deletion of MAC1 from previous studies [3]. Unlike macl strains, acil strains showed no change in vegetative growth, germination or conidiation. While both macl and acil mutants were affected in appressorium formation, the effect was more dramatic in macl mutants, which were unable to form any appressoria in 48 hours. Appressorium formation was significantly delayed in acil strains, however, difference in the number of mature appressoria at $24 \mathrm{hrs}$ were only modest. In addition macl strains were nonpathogenic whereas no change in virulence was found in acil strains. Overall, these results were rather surprising, especially after reviewing expression data from EST analyses as well as recent microarray experiments (GEO Accession: GES1945). ACI1 was found to be highly expressed under appressorium-inducing conditions as 19 ESTs were identified in the appressorium ESTs while no corresponding ESTs were identified in the mycelial ESTs [10]. Microarray data also showed the expression level of $A C I 1$ was high during germination and in immature appressoria. ACII was also found to be expressed in un-germinated conidia in microarray experiments. This is consistent with the finding that several $A C I 1$ ESTs were present in conidial specific EST library [10]. Thus, the generally high level of expression of $A C I 1$ would suggest that this gene plays an important biological role.

Due to the high level of expression and its predicted cellular location, we investigated whether the ACI1 mediates other pathways or processes. Specifically, we addressed the role ACI1 may play in osmotic stress sensing and modifying cell wall properties. In fungi, the HOG (high-osmolarity glycerol) pathway controls responses to osmotic shock [22]. In $S$. cerevisiae, the HOG pathway can be activated by MAPKK Pbs2 via signals from either of two yeast transmembrane osmosensors, Sho1 and $\mathrm{S} \ln 1$. Sho1 activate Pbs2 through Ste11 (MAPKKK) [23] while Sln1 activate Pbs2 via Sln1-Ypd1-Ssk1 multi-step phospho-relay system [24-26]. However, ACI1 does not show homology to either Shol or Sln1. Moreover, in our experiments, ACI1 does not appear to be required for growth in high salt conditions.

Hydrophobins, which form a rodlet layer in the cell wall, confer surface hydrophobicity. Disruption of the well characterized hydrophobin gene MPG1 in M. oryzae results in absence of the rodlet layer and the production of a watersoaked, easily wettable phenotype [27]. The phenotype is commonly associated with several other characterized fungal hydrophobins such as $\operatorname{rod} A$ and EAS genes of Aspergillus nidulans and $N$. crassa, respectively [18-20]. ACI1 contains a predicted signal peptide and a predicted extracellular CFEM domain, a conserved motif consisting of eight conserved cysteines similar to that found in hydrophobins although the spacing of cysteines was found to be different. However, the easily wettable phenotype was not associated with deletion of $A C I 1$. Thus, ACI1 does not appear to have properties found in other hydrophobin proteins.

In summary, disrupting $A C I 1$ led to subtle phenotypic changes of rice blast fungus in its ability to appressoria, although no differences were observed for disease symptoms, 
asexual development and vegetative growth under our tested conditions. The lack of distinctive phenotype typical of null mutants of other identified cAMP component genes may be due to functional redundancy. ACI1 may influence initial appressorial formation, but functionally redundant or other gene(s) such as $I M R$ and/or XP 364725 maybe able to compensate for the loss of ACI1. IMR and ACII exhibit a very similar pattern of gene expression. $I M R$ is actively expressed in conidia, germ tubes and during appressorium formation but not in vegetative and infection hyphae growth stages [21]. Furthermore, both imr and acil mutants failed to show clearly detectable alterations in virulence. As described above, XP 364725 is more closely related to ACII than IMR based on BlastP as well as the inferred phylogenetic tree. This gene also appears to be dispensable for appressorium formation and pathogenicity (Jun and Dean, unpublished data). It is thus possible that any 2 of the three genes may be able to compensate for the loss of another. However, redundant function does not necessarily require strong sequence homology. For example, several hydrophobins from different fungi can complement the $m p g 1$ phenotype defect where the sequence of these hydrophobins and MPG1 is very dissimilar [6]. Thus identifying possible functional redundant genes of $A C I 1$ assuming functional redundant gene or genes account for the observations in this study is challenging. However, it may be enlightening to evaluate double and triple gene knockout mutants.

\section{ACKNOWLEDGEMENT}

The authors would like to thank Dr. Yeon Yee Oh and Dr Junichiro Marui in Fungal Genomics Lab of North Carolina State University for the technical help and discussions. This work was supported by United States Department of Agriculture Cooperative State Research, Education and Extension Service and the National Science Foundation.

\section{REFERENCES}

[1] Howard RJ, Valent B. Breaking and entering: host penetration by the fungal rice blast pathogen Magnaporthe grisea. Annu Rev Microbiol 1996; 50: 491-512.

[2] Xiao JZ, Watanabe T, Kamakura T, Ohshima A, Yamakuchi I. Studies on cellular differentiation of Magnaporthe grisea. Physicochemical aspects of substratum surfaces in relation to appressoria formation. Physiol Mol Plant Pathol 1994; 44: 227-36.

[3] Choi W, Dean RA. The adenylate cyclase gene MAC1 of Magnaporthe grisea controls appressorium formation and other aspects of growth and development. Plant Cell 1997; 9(11): 1973-83.

[4] Mitchell TK, Dean RA. The cAMP-dependent protein kinase catalytic subunit is required for appressorium formation and pathogenesis by the rice blast pathogen Magnaporthe grisea. Plant Cell 1995; 7(11):1869-78.

[5] Talbot NJ, Kershaw MJ, Wakley GE, De Vries O, Wessels J, Hamer JE. MPG1 encodes a fungal hydrophobin involved in surface interactions during infection-related development of Magnaporthe grisea. Plant Cell 1996; 8(6): 985-99.

[6] DeZwaan TM, Carroll AM, Valent B, Sweigard JA. Magnaporthe grisea pth $11 \mathrm{p}$ is a novel plasma membrane protein that mediates appressorium differentiation in response to inductive substrate cues. Plant Cell 1999; 11(10): 2013-30.
[7] Liu S, Dean RA. G protein alpha subunit genes control growth, development, and pathogenicity of Magnaporthe grisea. Mol Plant Microbe Interact 1997;10(9): 1075-86.

[8] Kulkarni RD, Thon MR, Pan H, Dean RA. Novel G-proteincoupled receptor-like proteins in the plant pathogenic fungus Magnaporthe grisea. Genome Biol 2005; 6(3): R24.

[9] Talbot NJ. On the trail of a cereal killer: Exploring the biology of Magnaporthe grisea. Annu Rev Microbiol 2003; 57: 177-202.

[10] Kulkarni RD, Dean RA. Identification of proteins that interact with two regulators of appressorium development, adenylate cyclase and cAMP-dependent protein kinase A, in the rice blast fungus Magnaporthe grisea. Mol Genet Genomics 2004; 270(6): 497-508.

[11] Dobbeling U, Boni R, Haffner A, Dummer R, Burg G. Method for simultaneous RNA and DNA isolation from biopsy material, culture cells, plants and bacteria. Biotechniques 1997; 22(1): 88-90.

[12] Liu ZM, Kolattukudy PE. Early expression of the calmodulin gene, which precedes appressorium formation in Magnaporthe grisea, is inhibited by self-inhibitors and requires surface attachment. J Bacteriol 1999; 181(11): 3571-7.

[13] Carroll AM, Sweigard JA, Valent B. Improved vectors for selecting resistance to hygromycin. Fungal Genet Newslett 1994; 41: 22.

[14] Leung H, Lehtinen U, Karjalainen R, et al. Transformation of the rice blast fungus Magnaporthe grisea to hygromycin B resistance. Curr Genet 1990; 17(5): 409-11.

[15] Sambrook J, Fritsch EF, Maniatis T. Molecular Cloning: A Laboratory Manual. Cold Spring Harbor, NY: Cold Spring Harbor Laboratory Press 1989.

[16] Poirot O, O'Toole E, Notredame C. Tcoffee@igs: A web server for computing, evaluating and combining multiple sequence alignments. Nucleic Acids Res 2003; 31(13): 3503-6.

[17] Kulkarni RD, Kelkar HS, Dean RA. An eight-cysteine-containing CFEM domain unique to a group of fungal membrane proteins. Trends Biochem Sci 2003; 28(3): 118-21.

[18] Bell-Pedersen D, Dunlap JC, Loros JJ. The Neurospora circadian clock-controlled gene, ccg-2, is allelic to eas and encodes a fungal hydrophobin required for formation of the conidial rodlet layer. Genes Dev 1992; 6(12A): 2382-94.

[19] Lauter FR, Russo VE, Yanofsky C. Developmental and light regulation of eas, the structural gene for the rodlet protein of Neurospora. Genes Dev 1992; 6(12A): 2373-81.

[20] Stringer MA, Dean RA, Sewall TC, Timberlake WE. Rodletless, a new Aspergillus developmental mutant induced by directed gene inactivation. Genes Dev 1991; 5(7): 1161-71.

[21] Banno S, Kimura M, Tokai T, et al. Cloning and characterization of genes specifically expressed during infection stages in the rice blast fungus. FEMS Microbiol Lett 2003; 222(2): 221-7.

[22] Dixon KP, Xu JR, Smirnoff N, Talbot NJ. Independent signaling pathways regulate cellular turgor during hyperosmotic stress and appressorium-mediated plant infection by Magnaporthe grisea. Plant Cell 1999; 11(10): 2045-58.

[23] Posas F, Saito H. Osmotic activation of the HOG MAPK pathway via Ste11p MAPKKK: scaffold role of Pbs2p MAPKK. Science 1997; 276(5319): 1702-5.

[24] Maeda T, Takekawa M, Saito H. Activation of yeast PBS2 MAPKK by MAPKKKs or by binding of an SH3-containing osmosensor. Science 1995; 269(5223): 554-8.

[25] Maeda T, Wurgler-Murphy SM, Saito H. A two-component system that regulates an osmosensing MAP kinase cascade in yeast. Nature 1994; 369(6477): 242-5

[26] Posas F, Wurgler-Murphy SM, Maeda T, Witten EA, Thai TC, Saito H. Yeast HOG1 MAP kinase cascade is regulated by a multistep phosphorelay mechanism in the SLN1-YPD1-SSK1 "two-component" osmosensor. Cell 1996; 86(6): 865-75.

[27] Talbot NJ, Ebbole DJ, Hamer JE. Identification and characterization of MPG1, a gene involved in pathogenicity from the rice blast fungus Magnaporthe grisea. Plant Cell 1993; 5(11): 1575-90. 